\title{
Redes sociales y consumidores: participación y construcción de imagen
}

\author{
$\mathrm{M}^{\mathrm{a}}$ Henar Alonso MosQuera \\ Universidad CEU San Pablo \\ hemosquera.fhum@ceu.es \\ Ángel BARTOLOMÉ MuÑoz DE LunA \\ Universidad CEU San Pablo \\ abartolome@ceu.es
}

\begin{abstract}
Resumen:
La presente investigación analiza el fenómeno "fan" en Facebook como una forma de manifestación de los usuarios de sus intereses, aficiones y personalidad, y cómo a través de dicha adscripción a unos $\mathrm{u}$ otros grupos los consumidores participan junto a otros usuarios en la construcción de la imagen de marca.

El estudio ha trabajado sobre los perfiles oficiales de marcas renombradas, a los cuales se han adherido los estudiantes universitarios que componen la muestra. Se prefirió el análisis de dicho público por la familiaridad y el vínculo existente entre éste y las tecnologías de la información y la comunicación, como herramientas que emplea para comunicar y compartir. Así, a partir de la exploración de los perfiles oficiales de los que los usuarios eran seguidores, se han analizado las marcas y categorías de producto a las que pertenecen, para conocer cuáles son los sectores con mayor calado en las preferencias del público
\end{abstract}

Palabras clave: Facebook; Redes sociales; Investigación de mercados; Imagen corporativa; Marca; Fan.

\section{Social networks and consumers: voice and image building}

\begin{abstract}
:
This paper focuses on the "fan " phenomenon on Facebook as the form used by consumers to express their interests, hobbies and personality, and how through such detachment to them, users participate in the process of building brand identity.

This research is based on a survey between college students that analyzes the official profiles of wellknown brands they follow. Studying college students behavior was preferred as this is the audience with a more developed familiarity with information and communication technologies as tools used to communicate and share contents. After exploring the official profiles users were following, we have analyzed the brands and product categories to which they belong, to understand which are the ones with the greatest draft in public preferences
\end{abstract}

Key Words: Facebook; Social Media; Market Research; Corporate; Brand identity; Fan.

\section{Referencia normalizada:}

Alonso Mosquera, M. H. y Bartolomé Muñoz de Luna, A. (2014): Redes sociales y consumidores: participación y construcción de imagen. Historia y Comunicación Social. Vol. 19. Núm. Especial Febrero. Págs. 211-221. 
Sumario: 1. Facebook, un nuevo entorno de comunicación para las marcas 2. Metodología del estudio 3. Datos obtenidos 4. Conclusiones y discusión 5. Bibliografía

\section{Facebook, un nuevo entorno de comunicación para las marcas}

La emergencia de las redes sociales no supone sino una mera herramienta tecnológica al servicio de lo que el ser humano viene haciendo desde siempre: compartir e intercambiar sus ideas sus ideas y sentimientos como ser social que es. Así, el mundo online se adapta al comportamiento humano en el offline (Adams, 2012: 27), amplificando, eso sí, las posibilidades de comunicación disponibles.

Asimismo, los consumidores necesitan compartir sus ideas, dudas, alegrías y decepciones respecto de marcas y productos. Por ello, y como diversos estudios demuestran, cada vez más se utilizan las redes sociales para buscar información comercial, pues dichas comunicaciones son percibidas como menos intencionadas y más imparciales que las informaciones que vienen directamente de las empresas. Así, dichas redes juegan un papel de agentes de cambio fundamental en la forma en que concebimos la comunicación comercial. Conocer cómo personas con intereses comunes interaccionan y hablan de marcas y productos, será una fuente de información tan relevante como pueden ser la que nos proporcionan otras técnicas de investigación social, como los grupos de discusión o las encuestas. Por ello, los anunciantes deben replantear sus estrategias de comunicación en el mundo digital, considerando que sus mensajes deben adaptarse a un nuevo entorno colaborativo y bidireccional, donde las nuevas plataformas son las redes sociales, los espacios de microblogging o los canales de vídeo digital (Castelló, 2010), y donde "los mercados son conversaciones", como se afirmaba en el Manifiesto Cluetrain.

En Facebook, los grupos creados por los consumidores o las empresas proporcionan el foro idóneo para que pequeñas comunidades de personas con un interés común puedan comunicarse y expresarse en relación con dicho interés. Cualquier persona puede crear un grupo, e interactuar con otros usuarios, pero también existe la posibilidad de que empresas, organizaciones, famosos, marcas y productos puedan comunicarse con los consumidores que desean seguirles. Así, estos grupos formarían parte de lo que los sociólogos denominan "lazos débiles" con los que interactuamos debido a algún interés u objeto común (Adams, 2010:85), pero que pueden ser una fuente de información tan valiosa como los lazos fuertes (Granovetter, 1973).

La generación de estudiantes universitarios actuales forma parte de la denominada Generación Y. También conocida como C -"Creatividad, Contenido, Compartir"(Búa, 2010), "Echo Boomers" (OMD, 2012), "Net generation" (Oblinger y Oblinger, 2005) o generación ND (Nacido Digital), que está constituida en España por más de ocho millones de jóvenes. Es un usuario fuerte de redes sociales, y emplea las mismas para sentirse unido a los demás, y recibir y repartir conocimiento, al compartir sus experiencias y opiniones en redes que multiplican exponencialmente sus posibilidades de ser apoyado, reconocido, y retransmitido (Tabernero et al., 2010). Así, el 
interés de las marcas es aún mayor, pues la la familiaridad de los nativos digitales con las nuevas tecnologías, y muy especialmente con las redes sociales, hace amigables y cercanas las comunicaciones recibidas por este medio (Alonso y Bartolomé, 2011) y favorece la satisfacción de necesidades sociales de dichos jóvenes (Colás, González y Pablos, 2013), que las usan para interactuar con sus iguales y sentirse acogidos en la comunidad, priorizando las nuevas formas de comunicación frente a las basadas en el contacto personal.

Algunos datos cuantitativos que refuerzan estas teorías son los siguientes: el 93\% de los internautas comprendidos entre los 14 y los 17 años son usuarios habituales de las redes sociales (The Cocktail Analysis, 2013). El Séptimo Observatorio de Tendencias Nokia (2012) revela que el 64\% de los jóvenes entre 19 y 24 años utilizan Internet en el móvil, y nueve de cada diez utiliza las redes sociales -más de la mitad lo hace diariamente-. Aunque entre los más jóvenes, Facebook también es la plataforma más conocida y utilizada -como señalan Túñez y Sixto (2012), es la segunda página web más visitada, y casi el $90 \%$ de los usuarios de redes sociales tienen perfil en ella-, su red social favorita es Tuenti, según el estudio del IAB (IV Estudio Anual de Redes Sociales IAB-Elogia, 2013), con Twitter creciendo como "la red de moda" ((The Cocktail Análisis, 2013). En su uso de dichas redes, los jóvenes simultanean esta actividad con otras tareas como escuchar música $(82 \%)$, ver la televisión $(47 \%)$, estudiar (35\%), y otras como realizar trabajos de clase con baja implicación, ver películas o series en Internet, o hacer diversas tareas del hogar (11\%) (Monge y Olabarri, 2011: 94).

Algunos estudios contribuyen a esta idea de que las redes sociales son un vehículo más para la comunicación comercial y que participan así decisivamente en las decisiones de compra de sus usuarios. De hecho, se afirma que hasta el sesenta por ciento de los consumidores modificaría una intención de compra si encontrase comentarios negativos en las redes sociales respecto de dicha marca o producto (Forrester y Ernst $\&$ Young, 2012) y podría hablarse incluso de un modelo de compra colaborativa basada en la interacción de usuarios entre ellos y con las marcas (Brandreportblog, 2012). Por ello, toda empresa que desee interactuar con sus usuarios debe "estar ahí", participar del diálogo que en la red se establece para contribuir al proceso de engagement. En este sentido, la relación entre usuarios de las redes sociales pasa de ser vertical a ser horizontal, posibilitando una aparente igualdad, dado que el usuario puede actuar como emisor, transmisor o receptor de la información (Caldevilla, 2010).

Este nuevo consumidor y usuario de los medios sociales, cada vez más sofisticado en sus interacciones (The Cocktail Análisis, 2012), adquiere nuevos perfiles de acuerdo con las acciones que realiza en relación con sus marcas preferidas. Es un consumidor profesionalizado, prosumer, que considera y valora gran cantidad de información antes de tomar una decisión de compra(Castelló, 2010). Es fan incondicional de sus marcas favoritas, fansumer, por encima de su adscripción a ideologías políticas o religiosas (Búa, 2010), y además deja de ser un número más, cada consumidor tiene una personalidad, y puede hablarse del persumer, la combinación de persona y consumidor (Huertas, 2010). Así, puede considerarse que el consumidor 
presta atención de forma voluntaria a las acciones comerciales que las marcas realizan (García Guardia y Núñez, 2009), pero siempre respecto de aquellas marcas que son para él seña de identidad y que contribuyen a reforzar su personalidad y su estilo de vida (Miralles, 2008).

\section{Metodología del estudio}

El objetivo del presente estudio es mostrar cualitativamente la relación establecida entre jóvenes y marcas a través de las redes sociales, y más concretamente, en función de su presencia y seguimiento en Facebook. Dado que el proyecto se encamina a detectar las cualidades y carencias de las marcas y productos de consumo, de acuerdo con las preferencias expresadas por los usuarios, se han analizado las páginas y grupos creados en Facebook por los usuarios y por las propias empresas.

Esta primera parte del estudio, de carácter exploratorio, se centra en el análisis de los principales grupos y perfiles a los que se afilian en la red social los universitarios componentes de la muestra, con especial interés en el análisis de las categorías de los productos o marcas con los que desean mantener esta vinculación. Dado el perfil de usuario habitual de las redes sociales, respecto de rasgos demográficos y psicográficos, consideramos que entre nuestros propios estudiantes se podía establecer la mejor muestra de estudio de las posibles.

Así pues, a cada uno de los estudiantes que voluntariamente participó en el proyecto se le solicitó, en primer lugar, que cumplimentara una ficha en la que debía consignar los nombres de los grupos y páginas de las cuales eran seguidores en Facebook, pero solamente de aquellos que incluyesen en su denominación el nombre de una empresa o marca comercial. En segundo lugar, con el fin de ampliar la muestra objeto de estudio, y favorecer la participación garantizando la confidencialidad de los datos, se pidió a cada participante que consignase una ficha con los mismos datos de otros nueve estudiantes universitarios, seleccionados aleatoriamente de entre sus amigos en la red y para evitar duplicidades, se les indicó que no podían incluir a personas que fuesen también amigos en dicha red social de otros participantes en el estudio. Así, no podía saberse qué datos correspondían al voluntario y cuáles a sus amigos. Con todo ello, no lográbamos una muestra representativa estadísticamente, pero sí lo suficientemente significativa como para a partir de los datos obtenidos desarrollar una segunda fase del estudio.

A pesar de la absoluta confidencialidad de los datos (solo tenían que indicar edad, sexo, carrera cursada, y su listado de grupos), se creó un modelo de autorización para que los estudiantes recabasen la firma de aquellos amigos participantes en el proyecto, y de hecho, a la hora de tabular los datos, se desecharon aquellos provenientes de personas que no hubieran autorizado que sus datos formasen parte de la muestra. Una vez recibidas todas las fichas válidas y autorizadas, tabulamos los resultados correspondientes a los ochenta estudiantes participantes, veintiún hombres y 
cincuenta y nueve mujeres, todos ellos estudiantes universitarios entre veinte y veinticuatro años, y matriculados en hasta doce titulaciones distintas. Al haber solicitado a nuestros estudiantes que recurriesen a sus amigos, se evitaba el sesgo propio de acudir a estudiantes solo de Comunicación, que a priori y por nuestra experiencia son más activos en el mundo online.

Al tabular los datos procedentes de todos los participantes, finalmente recabamos un total de 611 grupos y perfiles distintos en Facebook de distintas marcas comerciales, tanto perfiles oficiales (creados por la propia empresa) como otros creados por usuarios seguidores o detractores de las mismas, y los clasificamos teniendo en cuenta, además de dicho criterio de oficialidad, la categoría de producto a la que se hace referencia, valor o connotación transmitida (lo que el nombre del grupo indica respecto del producto o marca al que hace referencia), y valoración de dicha connotación (positiva, negativa o neutra). Con toda esta información, se procedió al análisis de los resultados.

\section{Datos obtenidos}

La primera subdivisión realizada al analizar los datos recabados viene dada por el interés en separar por un lado aquellos grupos o perfiles en Facebook creados por usuarios particulares y por otro lado, los perfiles oficiales creados por las propias marcas. En este sentido, la mayor parte de los grupos analizados lo eran de carácter oficial, un total de 518 .

Así, la siguiente subdivisión es la referida a las categorías de producto con las que se vinculan las marcas seguidas por los estudiantes encuestados. A priori, podríamos considerar que los grupos o perfiles a los que los estudiantes se afilian están vinculados a las marcas o productos que son más importantes en su vida diaria. Analizando las marcas obtenidas, las agrupamos en las siguientes categorías y subcategorías: ocio, que comprende deportes, música, restauración, turismo, bebidas alcohólicas y ocio nocturno; alimentación y bebidas; moda (que engloba textil, belleza y bisutería); tecnología (desagregada en tecnología general, vídeo, móviles, juegos online y redes sociales); medios de comunicación y cultura (compuesta por televisión, cine, prensa, radio, revistas, libros y otros medios ); distribución comercial, otros productos (donde incluimos otras categorías con menor índice de seguimiento como automóviles y medicamentos) y otros servicios, que engloba banca, educación, grupos políticos, asociaciones y ONGs. De acuerdo con esta subdivisión, destacamos los datos más significativos de cada categoría y las marcas incluidas, mencionando otros criterios de clasificación en las conclusiones por disponer de un espacio limitado para comentar los aspectos más llamativos del estudio.

El veintidós por ciento de los grupos detectados se encuadran en la categoría de ocio. Dentro de ésta, podemos citar 37 grupos distintos vinculados al mundo del deporte, el 4,5\% del total, tanto grupos oficiales de clubes deportivos (Real Madrid, 
Atlético de Madrid, Barcelona o L.A. Lakers), o federaciones (NBA) como de deportistas de élite (Rafa Nadal, Cristiano Ronaldo, Messi o Usain Bolt), así como de marcas relacionadas con el mundo del deporte como Nike y Adidas.

Dos de cada tres usuarios encuestados son seguidores en redes sociales de algún grupo o perfil vinculado al mundo de la música, tanto de los perfiles de cantantes y grupos (Pereza, Red Hot Chili Peppers y The Kooks, los más seguidos) como de determinados eventos o festivales, entre los que destaca el seguimiento de Monegros y Creamfields. Respecto del mundo de la restauración, casi el setenta por ciento de los usuarios son seguidres de algún establecimiento, especialmente de aquellos de comida rápida y perfil más económico (seguramente, más propios del perfil de edad y poder adquisitivo al que nos hemos dirigido) como Ginos, Starbucks, Vips, Foster's Hollywood o Telepizza. Por último, y en la subcategoría de "turismo", encontramos 17 perfiles oficiales de aerolíneas (Binter, Iberia o Vueling y Vueling people), de lugares (Visit Argentina o Islas Canarias) o de bienes y servicios vinculados con el turismo (Guía Repsol, Rumbo, eDreams...).

La subcategoría de bebidas alcohólicas arroja como resultado en primer término que hasta el cuarenta por ciento de los encuestados son seguidores de alguna marca de esta tipología de productos. Así, encontramos 24 grupos distintos, de ellos los once de perfiles oficiales de los rones Santa Teresa, Brugal y Barceló, las cervezas Estrella Galicia, Carslberg, Amstel y Heineken, y asimismo de Tío Pepe, Martini, Jagermeister y la ginebra Beefeater. Un dato que llamó nuestra atención es que, pese al bajo coste que puede tener realizar acciones de comunicación en redes sociales, y la cercanía con los usuarios, al analizar el seguimiento de los perfiles de locales de ocio nocturno, detectamos que apenas un veinte por ciento de los estudiantes era seguidor de la página de alguno de ellos, y sin que ningún grupo concreto tuviera un seguimiento significativo.

Respecto del seguimiento de marcas de alimentación y bebidas, uno de cada cuatro participantes en el estudio reconoció ser fan de alguna de las marcas de la categoría. El cuatro por ciento de los grupos analizados se encuadra en esta categoría, y de ellos, la mayoría hacen referencia a dulces, aperitivos y snacks. Plátano de Canarias, Pringles España, Kinder Bueno, Ferrero Rocher, Nespresso, Suchard, NESCAFÉ España, Lindt Chocolate España, Conguitos, Carbonell, Oreo, Nutella, Häagen Dasz, After eight, Schweppes, Coca Cola y Pepsi son las marcas elegidas. Ninguna marca destacó por un seguimiento masivo, pero nos resultó especialmente sorprendente el bajo seguimiento en la muestra analizada de las marcas de bebidas refrescantes, pues solo el cuatro por ciento de los encuestados era seguidor de alguna de ellas, a pesar de la alta preferencia de marca y simpatía por las mismas que se desprende de otros estudios realizados sobre un target similar.

La segunda categoría con mayor número de seguidores, pues representa el veinte por ciento de los grupos analizados, es la referida a productos de moda. En este segmento, en primer término detectamos hasta 56 grupos vinculados al área de textil y complementos, el once por ciento del total. Entre las marcas seguidas por los estu- 
diantes, aparecen algunas de carácter más aspiracional como Chanel, Loboutin o Jimmy Choo, pero los que agrupan a mayor número de fans son los perfiles oficiales de marcas más asequibles y cercanas al perfil de compras de la muestra, como Abercrombie\&Fitch, Zara, Topshop, H\&M, Victoria's Secret, Asos o Bimba\&Lola. Igualmente, en la categoría de belleza se registran el cuatro por ciento de los grupos del estudio, pero solo tienen un número relevante de seguidores los referidos a productos o establecimientos de compra masiva, como son Pantene, L'Oreal, Sephora, Makeup Zone y Kiss\&Makeup. No hay datos destacados en la categoría de bisutería, y solo hay un seguimiento mínimo de las marcas Pandora y Aristocrazy.

El catorce por ciento de los perfiles seguidos hace referencia a productos vinculados con los medios de comunicación. Entre ellos, hasta treinta y tres páginas oficiales de series y programas de televisión, con Gossip Girl como página favorita (seguida por el $8 \%$ de los estudiantes). Es menos significativo el seguimiento de las subcategorías de cine, prensa, radio y libros, de más a menos seguidores, pero sí hay mayor preferencia por los perfiles en el ámbito de las revistas especializadas, donde uno de cada cuatro estudiantes sigue a alguna publicación periódica como Vogue, Telva, Cuore, Harpers Bazaar, Elle, GQ, Aletheia y The Arquitech Newspaper. Es destacable que hasta el trece por ciento de los encuestados es seguidor de alguna de las ediciones (nacional o extranjera) de la revista Vogue. Respecto de medios online, encontramos doce grupos referidos a blogs de moda y ocio, pero ninguno de ellos con un número significativo de seguidores.

Respecto al ámbito de la tecnología, el seis por ciento de los perfiles seguidos en el estudio encuadra en esta categoría de productos. Dentro de ella detectamos empresas vinculadas a las compras colectivas (Sorteamus, Easybuy, Amazon buy vip, Groupon, Privalia, Groupalia o Lets Bonus), sitios web de redes sociales y juegos en red (Filmaffinity, Blogger, Facebook, Farm Ville, Texas HoldEm Pocker o Angry Birds), fabricantes de dispositivos y prestadores de servicios vinculados al mundo online como Apple, Orange, Iphone, Blackberry, Samsung Polaroid, iTunes, iPoduplink o Intel, y de forma muy destacada, fans de páginas de descarga gratuita de contenidos de ficción, con Series.ly en cabeza, seguida por el 14\% de los encuestados.

Otras categorías incluídas en el estudio, que se consideraron como posibles focos de interés pero que al analizar la muestra arrojaron unos datos de seguimiento muy bajo, y por tanto se agruparon en "otros productos" son automoción, con apenas cuatro grupos mencionados, dos perfiles de especialidades farmacéuticas, cinco grupos en la categoría de distribución comercial de productos de gran consumo, y por último en servicios, mencionamos de forma anecdótica a los dos perfiles de banca detectados (Openbank y La Caixa, con un único seguidor cada uno de ellos) y cuatro perfiles de entidades vinculadas al mundo de la educación. Por último, encontramos hasta nueve ONGs con un seguidor cada uno, y tres perfiles de carácter político, de nuevo con un único fan, que son los de UPyD, Esperanza Aguirre y Mariano Rajoy. 


\section{Conclusiones y discusión}

La investigación realizada es una primera parte de un estudio de mayor alcance sobre el seguimiento de marcas en redes sociales. Por ello, y como primera aproximación, el tamaño de la muestra analizada no permite establecer conclusiones válidas desde un punto de vista de representatividad estadística. No obstante, sí arroja datos interesantes a partir de los que establecer las hipótesis para el estudio posterior.

En primer lugar, y respecto del seguimiento de los perfiles oficiales de marcas, creados por las propias entidades, puede ser relevante en primer término separar el interés por marcas y productos actualmente consumidos por el perfil de usuario analizado, frente a otras marcas a las que siguen por su imagen positiva y de prestigio, y en este sentido, discernir el tipo de acciones de comunicación en redes sociales que debe desarrollar cada una de ellas. Así, frente a las acciones de marcas que consumen habitualmente, que podrían ser tanto de mejora de la imagen corporativa como acciones comerciales de carácter más puntual (informar de lanzamientos, participar en promociones, etc.), aquellas marcas de moda, belleza, bisutería o automoción, entre otras, que actualmente no son consumidas por nuestro target, pero a las que sigue en redes sociales con un carácter más aspiracional, pueden aprovechar esta relación para reforzar el valor de la marca y lograr la preferencia cuando estos usuarios puedan ser consumidores de la misma. Hay que tener en cuenta que los jóvenes universitarios, aunque encajan en el perfil de usuarios fuertes de las redes sociales, no tienen la renta necesaria para ser consumidores de determinados productos que seguramente puedan comenzar a consumir en el futuro, cuando su renta sea más elevada.

Dada esta limitación para el consumo, puede ser interesante enfocar estudios posteriores a jóvenes adultos, que por franja de edad son también usuarios fuertes de redes sociales, pero como consumidores presentan un perfil de gasto y una capacidad adquisitiva sensiblemente mayor.

Como ya se ha mencionado, el seguimiento de marcas que actualmente consume nuestro público puede ser relevante para analizar qué tipo de acciones realiza en redes sociales, si hay relación entre el número de fans y las acciones desarrolladas, y con ello establecer una política de "buenas prácticas" en la relación con el mismo. Diversos productos y marcas relacionados con el ocio y tiempo libre, medios de comunicación, restauración, moda, alimentación y bebidas... en general, categorías que sí pueden realizar acciones promocionales dirigidas claramente a este target, deberían aprovechar la capacidad de las redes sociales para este fin.

Frente a marcas y categorías de producto que han obtenido un alto índice de seguimiento, encontramos otras con un interés muy bajo por parte del público estudiado. Al margen del carácter no científico de la muestra, sí es cierto que parece lógico que por edad, estilo de vida y nivel de renta los estudiantes universitarios no tengan mayor interés en determinados productos y servicios como pueden ser los relacionados con el sector de banca, seguros... que actualmente no están en su rango de consumo. Así, sería interesante comprobar si esta falta de afiliación se repite como 
patrón en públicos con un perfil de edad y gasto superiores -y por tanto, posible target de dichas compañías-, y si es así, conocer qué motivos ocasionan este bajo índice de seguimiento y analizar qué acciones de comunicación desarrollan dichas empresas en redes sociales para valorar si están haciendo lo correcto para captar seguidores.

Igualmente, es muy llamativo el desinterés que muestran los estudiantes participantes en el estudio por las organizaciones de carácter político, religioso o asistencial. Es cierto que otros estudios previos ya han puesto de manifiesto la falta de interés de la "generación Y" por dichas cuestiones, y su mayor interés y credibilidad percibida en los mensajes comerciales que les transmiten sus marcas preferidas. No obstante, sí sería interesante conocer hasta qué punto la falta de afiliación en las redes sociales a grupos de esta tipología responde a un desinterés real en sus vidas por dichas cuestiones, si las entidades que se encuadran en este tercer sector no están aprovechando las posibilidades de comunicación que los medios emergentes ofrecen, o si esta falta de seguimiento es meramente "virtual" y responde a un deseo de privacidad, en tanto en cuanto los usuarios no se afilian a aquellos perfiles que ponen de manifiesto antes sus contactos cuáles son sus ideas e inquietudes en temas sensibles como la ideología política o religiosa.

Asimismo, otros aspectos sobre los que se ha puesto de manifiesto que podríamos formular hipótesis de acuerdo con los datos obtenidos se vinculan a las preferencias en función del sexo, edad e incluso intereses profesionales (tomados en función de los estudios realizados) de los encuestados.

\section{Bibliografía}

ADAMS, Paul (2013): Grupos: la unión hace la fuerza en la red social. Social Business, Anaya. Madrid.

ALONSO, Henar y BARTOLOMÉ, Ángel (2011). Emprendedores y creativos: un concurso creativo apoyado en redes sociales para la docencia de publicidad y Relaciones Públicas, en VV.AA., La comunicación pública secuestrada por el mercado. Cuadernos artesanos de Latina, $\mathrm{n}^{\mathrm{o}}$ 14, pág. 278-315.

BARTOLOMÉ, Ángel, VIÑARÁS, Mónica y LLORENTE, Carmen (2012): Emprendedores y creativos: una propuesta interdisciplinar de innovación docente, en Revista de Comunicación Vivat Academia, no 117E. Disponible en: http:// pendientedemigracion.ucm.es/info/vivataca/numeros/n117E/DATOSS.htm\#81. Consultado 12 de junio de 2013.

BERNETE, Francisco. (2010). Usos de las TIC, relaciones sociales y cambios en la socialización de las y los jóvenes, en Revista de Estudios de Juventud, nº 88, pág. 97-114.

BRANDREPORTBLOG (2012). Generación Empowered: el comportamiento de compra de los millenials. Disponible en http://www.brandreportblog.com/ wpress/generacion-empowered-el-comportamiento-de-compra-de-los-millennials/. Consultado el 10 de diciembre de 2012. 
BROOKS, David (2011): El animal social: las fuentes secretas del amor, la personalidad y los logros. Ediciones B. Madrid.

BÚA, Sagrario (2010): La Generación C. Encuentro de Investigadores en Comunicación, Universidad CEU San Pablo, Madrid, 22 de junio.

CALDEVILLA, David (2010). Las redes sociales: tipología, uso y consumo de las redes 2.0 en la sociedad digital actual, en Documentación de las ciencias de la información, $\mathrm{n}^{\circ} 33$, pág. 45-68. Disponible en http://dialnet.unirioja.es/servlet/ aleart?codigo $=3250105$. Consultado el 12 de septiembre de 2013 .

CASTELLÓ, Araceli (2010): Crossumer, prosumer, fansumer y persumer. Estrategias empresariales en social media. Disponible en http://www.aracelicastello. com/2010/07/crossumer-prosumer-fansumer-y-persumer.html. Consultado el 13 de diciembre de 2012.

COLÁS, Pilar, GONZÁLEZ, Teresa y PABLOS, Juan (2013). Juventud y redes sociales: Motivaciones y usos preferentes, en Comunicar, $\mathrm{n}^{\circ}$ 40, vol. XX, pág. 15-23.

FORRESTER Y ERNST \& YOUNG (2012). Redes sociales. Nuevo juego, nuevas reglas, nuevos ganadores. Disponible en http://www.ey.com/Publication/ vwLUAssets/Redes_Sociales/\$FILE/Redes_Sociales.pdf. Consultado el 7 de mayo de 2013.

GARCÍA GUARDIA, María Luisa y NÚÑEZ, Patricia (2009). Los Bloggers y su influencia en la imagen de una marca. Revista Icono14, n 12 , pág. 242-252.

GRANOVETTER, Mark S. (1973). The strength of weak ties, en American Journal of Sociology, vol. 78, $\mathrm{n}^{\circ}$ 6, pág. 1360-1380. Disponible en http://www.jstor.org/ stable/2776392. Consultado el 5 de septiembre de 2013.

HUERTAS, Víctor Manuel (2010). The Next Best Brand. (o cómo los consumidores más innovadores conciben su relación con las marcas en el futuro escenario post-crisis). Seminario Aedemo/AEA. Disponible en http://download.aedemo.es/ aedemo/seminarioaea2010/huertas.zip. Consultado el 10 de mayo de 2013.

MIRALLES, Rafael (2008). Cultura juvenil, consumismo y medios de comunicación. Disponible en http://www.bduimp.es/archivo/conferencias/pdf/08_10152_10_ RafaelMiralles_idc9942.pdf. Consultado el 9 de febrero de 2013.

MONGE, Sergio. y OLABARRI, Elena. (2011): Los alumnos de la UPV/EHU frente a Tuenti y Facebook: usos y percepciones. Revista Latina de Comunicación Social, $\mathrm{n}^{\circ}$ 66. Disponible en: http://www.revistalatinacs.org/11/art/925_UPV/04_Monge. html. Consultado el 31 de octubre de 2012.

NOKIA (2012). Séptimo Observatorio de Tendencias Nokia. Los jóvenes, los móviles y la tecnología. Disponible en http://www.slideshare.net/conectarc/7-observatorio-de-tendencias-nokia-los-jvenes-los-mviles-y-la-tecnologa. Consultado el 25 de noviembre de 2012.

OBLINGER, Diana y OBLINGER, James (2005): It is age or IT: First step towards understanding the net generation, en OBLINGER, Diana y OBLINGER, James: Educating the net generation. Educause, Washington.

OMD (2012). Millenials. Disponible en http://www.omd.es/DigitalCenter/Onmedia/ Agosto12/3.html. Consultado el 5 de noviembre de 2012. 
TABERNERO, Carlos, ARANDA, Daniel y SÁNCHEZ, Jordi (2010). Juventud y tecnologías digitales: espacios de ocio, participación y aprendizaje, en Revista de Estudios de Juventud, $\mathrm{n}^{\circ}$ 88, pág. 77-96.

THE COCKTAIL ANALYSIS (2012): Informe público de resultados Observatorio Redes Sociales $4^{a}$ Oleada. Documento online. Disponible en http://www.slideshare.net/TCAnalysis/5-oleada-observatorio-redes-sociales. Consultado el 12 de mayo de 2013.

THE COCKTAIL ANALYSIS (2013): Informe público de resultados Observatorio Redes Sociales $5^{a}$ Oleada. Documento online. Disponible en http://www.slideshare.net/TCAnalysis/5-oleada-observatorio-redes-sociales. Consultado el 12 de mayo de 2013.

\section{Los autores}

$\mathrm{M}^{\mathrm{a}}$ Henar Alonso Mosquera es profesora de la Facultad de Humanidades y CC. de la Comunicación de la Universidad CEU San Pablo y Coordinadora del grado en Publicidad y Relaciones Públicas. Doctora por la Universidad CEU San Pablo, sus líneas de investigación se vinculan a las estrategias de comunicación y marketing online en el sector de gran consumo, y muy especialmente las desarrolladas para los niños y los jóvenes. Es miembro del Centro de Investigación en Tendencias de la Comunicación y participante en distintos proyectos y grupos de investigación.

Ángel Bartolomé Muñoz de Luna es profesor de la Facultad de Humanidades y CC. de la Comunicación de la Universidad CEU San Pablo y director del Máster Universitario en Diseño gráfico de la Comunicación Instituto de las Artes de la Comunicación TRACOR. Doctor en comunicación por la Universidad CEU San Pablo, licenciado en Filosofía por la Universidad San Dámaso y licenciado en publicidad por la Universidad CEU San Pablo y master de gestión de diseño en el EOI. Responsable de la Unidad Docente de Creatividad Publicitaria. Recientemente ha publicado, junto con otros autores, el libro "Los estudios de Comunicación en el EEES" y "La tecnología audiovisual al servicio de la sociedad". Así como varias publicaciones sobre la publicidad y creatividad en revistas especializadas. 